\title{
Green Synthesis of Silver Nanoparticles Using Valeriana officinalis Aqueous Extract
}

\author{
F. Mares-Briones ${ }^{1}$, M.S. Aguilar ${ }^{1}$, N. Fletes $^{1}$, S. E. Borjas-García ${ }^{2}$ and G. Rosas ${ }^{1}$ \\ 1. Instituto de Investigaciones en Metalurgia y Materiales, Departamento de Metalurgia Física, UMSNH \\ Morelia, Michoacán, México. \\ 2. Instituto de Física y Matemáticas, UMSNH, Morelia, Michoacán, México.
}

In recent years, the silver nanoparticles have attracted the interest of research in Nanoscience and nanotechnology due to its advantageous applications in biomedicine, drug delivery, food industry, agriculture, textile, water treatment, catalysis, antibacterial and anti-fungal [1]. Currently, green synthesis of nanoparticles has received enormous attention because it is clean, non-toxic and environmentally friendly method [2-3]. This work, exposes biosynthesis AgNPs by biochemical reduction of $\mathrm{Ag}^{+1}$ to $\mathrm{Ag}^{0}$ from silver nitrate solution $\left(\mathrm{AgNO}_{3}\right)$, using an aqueous extract of Valeriana (Valeriana officinalis) as a reducing agent and stabilizing agent at room temperature.

The V. officinalis root extract was milled to obtain fine powders to ensure more significant leaching of active substances. The extract was prepared with different concentrations of plant powder in $50 \mathrm{~mL}$ of distilled water $(0.25,0.50,0.75$ and $1.0 \mathrm{~g})$. Subsequently, reaction mixtures with each of the concentrations of the extract were prepared using the silver salt at $5 \mathrm{mM}$. The nanoparticles were characterized by UV-vis, XRD, SEM, and TEM. The color change of the aqueous extract from yellow to brown indicated the formation of silver nanoparticles in the reaction mixture. $10 \mathrm{~mL}$ aliquots of each the experiment were collected and analyzed by UV-vis spectroscopy. The appearance of an absorption peak between 420-450 nm, which is due to the presence of silver nanoparticles in solution [4], was observed. As concentration increases of the $\mathrm{V}$. officinalis extract the density of nanoparticles increased, keping constant the concentration of silver salt in $5 \mathrm{mM}$ (Fig. 1). SEM analysis confirmed the presence of silver nanoparticles in the reaction medium. Fig. 2 (a) shows polydispersed particles mostly of them spherical in nature and with particle sizes below $100 \mathrm{~nm}$. Fig. 2 (b) displays a dispersive energy analysis (EDS) confirmed the existence of silver in the solid state. Metallic silver shows a characteristic peak at approximately $2983 \mathrm{keV}$ [5]. X-ray diffraction pattern in Figure 3 shows the crystalline nature of the silver nanoparticles synthesized by aqueous V. officinalis extract. The diffractogram displays four different diffraction peaks observed in the range of $2 \theta ; 35-80^{\circ}$. Which can be indexed with the planes (111), (200), (220) and (311) of the face-centered cubic phase of silver, in agreeing with Joint Committee on Powder Diffraction Standards (JCPDS) data file [00-004-0783]. The size and morphology of the synthesized nanoparticles were determined by TEM images (Fig. 4). The shape of the synthesized nanocrystals was spherical. The size range of the nanoparticles is between 10-60 nm with an average size of $22 \mathrm{~nm}$. Higher density of nanoparticles is observed at a concentration of the plant extract of $1 \mathrm{~g}$ (Fig. 4a-b). Fig. 4c shows a high-resolution image (HR-TEM), and its corresponding FFT pattern reveals the crystalline nature of AgNPs. The inter-spacing corresponds to the $0.23 \mathrm{~nm}$ distance that belongs to (111) crystallographic planes of silver.

In summary, silver nanoparticles were synthesized by a biological method using an aqueous extract of Valeriana officinalis as reducing and stabilizing agent. UV-vis spectroscopy showed the SPR absorption band at $440 \mathrm{~nm}$, characteristic of AgNPs. The crystalline nature of the synthesized nanoparticles is evidenced by the diffraction peaks in XRD analysis. The morphological studies by electron microscopy show spherical AgNPs with an average size of $22 \mathrm{~nm}$. 
References:

[1] Abdolhossein Miri et al, Volume 141 (2015), p. 287.

[2] Shikuo Li et al, Green Chem. (2007).

[3] T. C. Prathna et al, B Biol. Sci 84 (2014), p. 65.

[4] Babak Sadeghi et al, Spectrochim acta A: Molecular and Biomolecular Spectroscopy 134 (2015), p. 310.

[5] Umesh B. et al, Ind Crop Prod 46 (2013), p. 132.

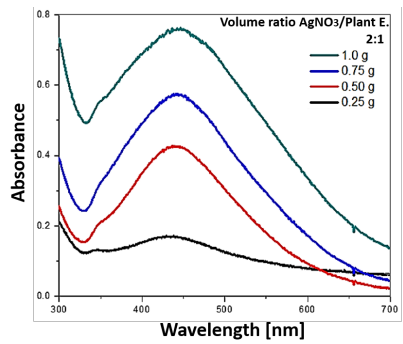

Figure 1. UV-Vis spectra of AgNPs synthesized at different plant extract concentration.

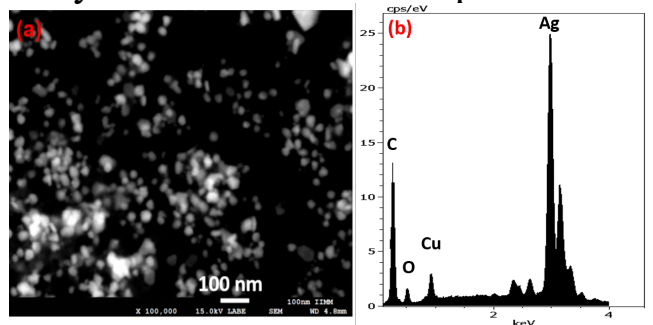

Figure 2. (a) SEM of silver nanoparticles obtained using Valeriana officinalis extract and (b) EDS analysis.

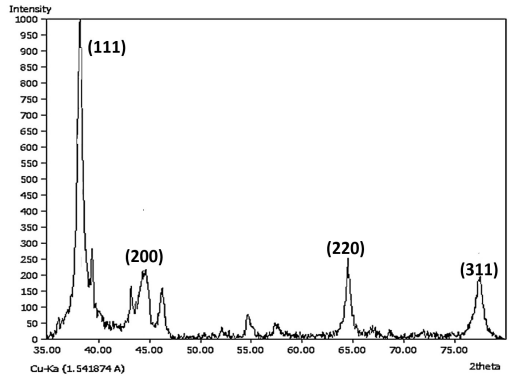

Figure 3. XRD pattern of silver nanoparticles biosynthesized.
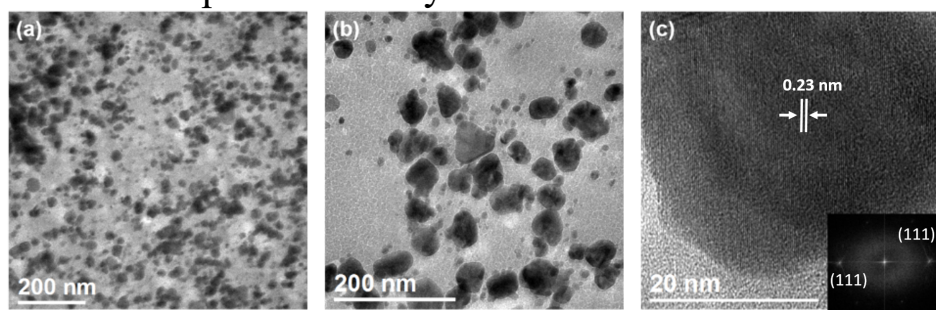

Figure 4. (a-b) Bright-field TEM images of AgNPs and (c) HR-TEM micrographs of individual AgNPs and their corresponding FFT pattern. 